Fecha de recepción: marzo 2020 Fecha de aceptación: abril 2020 Versión final: junio 2020

\section{Re-presentaciones de la imagen femenina en las series televisivas Las heroínas de Westword (2016-2018) de Jonathan Nolan y Lisa Joy}

Mónica Gruber ${ }^{(1)}$

Resumen: en 2016, la cadena HBO produjo para sus canales Premium la serie Westworld, creada por Jonathan Nolan y Lisa Joy. Esta serie de ciencia ficción y suspense nos transporta a un futuro cercano en el cual en uno de los parques temáticos creados por la empresa Delos Inc. se abre la posibilidad de vivir y experimentar el viejo Oeste americano o, en su segunda temporada, la alternativa de dos nuevas atracciones: Taj World y Shogun World. Los visitantes ("recién llegados" o "invitados") conviven con anfitriones (androides) creados para hacer posible esta aventura. Para ello se han pergeñado una serie de narrativas entrelazadas capaces de satisfacer todos los deseos de los huéspedes, preservando, sin embargo, la integridad física de los mismos. Es en este contexto en el cual dos de sus anfitrionas recuperan fragmentos de "memorias borradas" que las hacen poner en duda la veracidad de su mundo. Nos referimos a Dolores Abernathy, hija de un ranchero y Maeve Millay, madame del salón de Sweetwater Hall. Ellas se convertirán a lo largo de las dos temporadas en las auténticas heroínas de este relato serial.

Nos proponemos en este trabajo analizar las líneas hermenéuticas que dieron vida a estos personajes femeninos y cómo han sido desarrollados desde el punto de vista del lenguaje audiovisual. Creemos que no es casual que este tipo de narrativas emerja en momentos en los cuales el rol de la mujer en la sociedad es producto de profundas reflexiones políticas, éticas y estéticas.

Palabras clave: personajes femeninos - heroína - vida artificial.

[Resúmenes en inglés y portugués en las páginas 147-148]

(1) Mónica Gruber. Licenciada y Profesora en Artes (UBA). Profesora de la UP en el Área Audiovisual de la Facultad de Diseño y Comunicación. Profesora Adjunta, a cargo de La Literatura en las Artes Combinadas I / Literatura en las Artes Audiovisuales en Diseño de Imagen y Sonido (UBA) y Jefa de Trabajos Prácticos en la FFyL (UBA). Profesora del Museo Social Argentino, de la Universidad Tecnológica Nacional y de los Profesorados Artísticos del Gobierno de la Ciudad.

Directora del Proyecto PIA de Investigación Pervivencia y resemantización de los mitos en el mundo contemporáneo. De la narración oral a la pantalla global (FADU-UBA). Ha 
participado como ponente en Congresos Internacionales y Nacionales. Tiene publicados capítulos en volúmenes de la UP, de la Facultad de Filosofía y Letras, de FADU y de la Academia Nacional de Ciencias de Buenos Aires.

\section{Introducción}

Los siglos XX y XXI trajeron aparejados grandes cambios: se habló de la muerte de la historia, de la muerte del arte, de la muerte de los grandes relatos y hasta de la muerte del cine. Las pantallas, lejos de desaparecer, se hipertrofiaron y se multiplicaron exponencialmente. Las tecnologías electro-informáticas experimentaron una gran propagación. La aparición de la televisión por cable y su posterior desarrollo; la oferta de programación a la carta con opción a paquetes Premium a la medida de los consumidores; las plataformas de transmisión en streaming; los dispositivos electrónicos (smartphones, tablets, etc.), contribuyeron en esta era globalizada a su rápida expansión y consumo de todos los productos mediáticos. No obstante, si bien el mundo se expandía como resultado de ello, el aislamiento y la individualización se transformaron en las notas características de nuestro presente. Cada individuo vive pendiente de sus dispositivos electrónicos: (hiper) comunicado a partir de las posibilidades que le brinda el mundo tecnológico, pero aislado de sus pares y, muchas veces, incapaz de sostener vínculos presenciales.

No todas las modificaciones que hemos atravesado provinieron del campo digital o del electrónico: hemos asistido también a profundos cambios de perspectiva en el mundo social. El binarismo con el cual se concebía la sexualidad ha sido puesto en tela de juicio. Movimientos como el LGTBQI+ (de Lesbianas, Gays, Travestis, Transexuales, Transgénero, Bisexuales, Queer, Intersex, etc.) hicieron de la lucha contra la discriminación sexual su bandera ante la imposibilidad de verse representados en las anteriores estructuras patriarcales. Es en este marco que la "Tercera Ola Feminista" se ha expandido y cobrado un empuje inusitado en muchos países del mundo. El empoderamiento de la mujer es indiscutible. Los movimientos contra la violencia de género, las denuncias por violación y las marchas a favor del aborto, han sido solo algunas de las acciones emprendidas colectivamente que cobran cada vez más vigencia en las diversas sociedades.

Tal como hemos señalado, las grandes cadenas televisivas y las plataformas streaming han expandido su alcance a nivel global. Satisfacer la demanda de los millones de abonados con los que cuentan y sumar cada día potenciales clientes, los lleva a ofrecer una variedad de programas que se renueva permanentemente. Las series de televisión se han constituido en la fuerte apuesta de estos holdings de modo tal que podríamos afirmar, sin temor a equivocarnos, que nos hallamos transitando una época semejante a lo que fuese otrora la "Edad de Oro" del cine, solo que esta vez, de la televisión.

En 2016, HBO produjo para sus canales Premium la serie Westworld, creada por Jonathan Nolan y Lisa Joy. Esta serie de ciencia ficción y suspense nos transporta a un futuro cercano en el cual en uno de los parques temáticos creados por la empresa Delos Inc. se abre 
la posibilidad de vivir y experimentar el viejo Oeste americano o, en su segunda temporada, la alternativa de dos nuevas atracciones: Taj World y Shogun World. Los visitantes ("recién llegados" o "huéspedes") conviven con anfitriones (androides) creados para hacer posible esta aventura. Para ello se han pergeñado una serie de narrativas capaces de satisfacer todos los deseos de los huéspedes, preservando, sin embargo, la integridad física de los mismos. Es en este contexto en el cual dos de sus anfitrionas recuperan fragmentos de "memorias borradas" que las hacen poner en duda la veracidad de su mundo. Nos referimos a Dolores Abernathy, hija de un ranchero y Maeve Millay, madame del salón Sweetwater. Ellas se convertirán a lo largo de las dos temporadas en las auténticas heroínas de este relato serial.

Nos proponemos en este trabajo analizar las líneas hermenéuticas que dieron vida a estos personajes femeninos y cómo han sido desarrollados desde el punto de vista del lenguaje audiovisual. Creemos que no es casual que este tipo de narrativas emerja en momentos en los cuales el rol de la mujer en la sociedad es producto de profundas reflexiones políticas, éticas y estéticas.

\section{Westworld antes de Westwold}

En 1973 Michael Crichton escribió y dirigió el film homónimo, que fuera su ópera prima en el campo cinematográfico. En él, la compañía Delos ofrece una experiencia vacacional "cara y extraordinaria", tal como la publicitan, pudiéndose optar por visitar tres parques temáticos: el mundo del lejano oeste, el de la Edad Media y el tercero, la Roma imperial. La historia gira en torno a la creación de un parque de atracciones integrado por androides que terminan en un corto plazo asesinando a los humanos que visitan el predio.

Consideramos que el rol de las mujeres en esta narrativa se ve reducido a papeles secundarios o bien a la satisfacción de los placeres sexuales de los visitantes. De hecho, al comenzar a registrarse fallas en la programación, el personal señala con respecto a una anfitriona que: "se resiste a la reducción de un cliente".

Los androides son fácilmente identificables ya que "no lograron perfeccionar las manos". Entre los protagonistas hallamos a Richard Benjamin, James Brolin y Yul Brinner, éste último encarna a un despiadado pistolero androide.

A nivel técnico se utilizó por primera vez en un film el tratamiento digital $2 \mathrm{D}$ para tratar de representar la visión subjetiva de un ser de inteligencia artificial, recurriéndose a pixelar la imagen para emularla.

Cabe mencionar que el film fue nominado a los premios Saturno, Nébula y Hugo.

En 1976 Richard Heffron dirigía Futureworld, la secuela de de su predecesora. Crichton no estuvo vinculado esta vez al proyecto. El guion estuvo a cargo de Mayo Simon y George Schenk, pero el film no fue bien recibido por la crítica. Peter Fonda y Arthur Hill formaban parte del elenco y se incorporaba Yul Brinner encarnando al pistolero asesino, solo en una secuencia onírica. En este caso, la historia comenzaba un par de años después del desastre del parque; reconstruido, ya no ofrecía peligro para los seres humanos, salvo que detrás de la aparente reapertura se ocultaba un maquiavélico plan: la sustitución de 
los principales líderes por seres antropomorfos creados en forma sintética. Entre los recursos pioneros a nivel técnico se incluyó una secuencia diseñada en $3 \mathrm{D}^{1}$.

En 1980 sería el turno de la serie televisiva Beyond Westwold, creada por Michael Crichton, basada en el film de 1973. Compuesta de cinco capítulos, solo tres de ellos serían emitidos antes de su cancelación. A pesar de ello, fue nominada en las categorías Mejor Maquillaje y Dirección de Arte en los Primetime Emmy Awards. En este caso, John Moore (Jim McMullan) encargado de la Seguridad de Delos Corporation Inc. trata de detener a Simon Quaid (James Wainwright), un cinetífico que planea conquistar el mundo infiltrando a los androides que creó.

Finalmente, en 2016 será el turno de la aparición de la serie Westworld, creada por Jonathan Nolan y Lisa Joy. Basada en la película homónima de 1973 y en su secuela de 1976. Nolan y Joy junto con J. J. Abrams integran la producción de la serie. La primera temporada, "El laberinto", contó con diez capítulos; igual número para la segunda, titulada "El portal"; la tercera, cuyo estreno se produjo el 15 de marzo de 2020 contará con ocho capítulos reunidos bajo el nombre de "Nuevo Mundo".

\section{Un mundo del tamaño del dinero}

La serie cuenta con una compleja narrativa producto de varias líneas temporales que se imbrican, así como, numerosos personajes principales y secundarios que dan vida al relato.

Publicitado como la gran atracción turística para ricos, Westworld es el parque de diversiones inmersivas que permite experimentar aventuras únicas. Los huéspedes son recibidos por anfitriones -androides que son réplicas humanas perfectas, destinados a protagonizar las aventuras y satisfacer todos los deseos de los visitantes- que no amenazan la integridad física de los visitantes, pero que pueden ser destruidos tanto por sus semejantes como por los humanos.

El parque cuenta según se menciona unas cien tramas narrativas entrelazadas, monitoreadas desde la Mesa, una gran maqueta, ubicada en el edificio donde son creados y reparados los anfitriones. Tanto los seres antropomorfos como los animales son producto de desarrollos en máquinas impresoras 3D, laboratorios de conducta, de vestuario, reparación, morgue, etc. Los androides dañados son reparados, sus memorias son borradas, luego son reprogramados y devueltos al predio para garantizar el continuo flujo narrativo.

Al llegar al parque los visitantes eligen el atuendo que utilizarán durante su estadía y deben deshacerse de todas las pertenencias que los ligan al mundo exterior. Una vez ataviados resulta imposible discernir entre seres humanos y seres sintéticos. Cabe señalar que los anfitriones desconocen la existencia del sitio del que provienen los huéspedes. Los androides reviven cada día la misma narrativa, para ello se los repara, reprograma y se borra su memoria a diario como forma de asegurar su correcto y sistemático funcionamiento. Las atracciones de Delos -compañía creadora y dueña de las mismas- están recreadas en una isla en el medio del Océano Pacífico donde la empresa mencionada detenta el control y la soberanía. 
El lejano Oeste americano de la primera temporada de la serie es una "réplica" de Castle Valley, la legendaria ambientación de los westerns de John Ford, ya que se eligieron los espacios naturales de Utah (USA) para la filmación. Se incorporarán nuevos escenarios -el oriente japonés de la etapa Edo y la India en la época de la colonia inglesa- para la segunda. La tercera temporada, estrenada en Argentina el 15 de marzo de 2020 promete la incorporación del cuarto de los seis parques. Esta nueva ambientación transportará al espectador a la $\mathrm{II}^{\circ}$ Guerra Mundial, de los otros parques se desconoce todo dato. Hasta el momento se desconoce la narrativa que desarrollarán los faltantes durante el desarrollo de la cuarta temporada que ofrecerá la conclusión.

En los diez primeros capítulos, dos líneas narrativas temporales se cruzan constantemente: la apertura del parque -ubicada en nuestra época presente, en nuestra realidad-y la situación del parque treinta y siete años después -hacia 2052 aproximadamente-. Westworld ha sido producto de la creación de Robert Ford y su socio Arnold Weber. Este último ha muerto antes de la apertura de la atracción, en oscuras circunstancias, pero ha dejado escondidos en los anfitriones históricos una serie de códigos ocultos de programación que irán generando dificultades en las narrativas.

Dolores Abernathy, la primera anfitriona creada, lleva una tranquila vida hasta que su padre, presa de un ataque de locura luego de haber hallado una fotografía color y no poder identificar de qué se trata, le dice: “¡Debes irte! ¿No lo ves? ¡El infierno está vacío y todos los demonios están aquí!” Luego le susurra al su oído una frase inaudible. Dolores recuperará fragmentos de recuerdos de vidas pasadas que le fuesen arrebatados en múltiples operaciones de borrado de su memoria y comenzará a desarrollar conciencia. En línea con esto, casi en forma simultánea Maeve la matrona del salón Mariposa comenzará a recordar: a una hija de su pasada vida, así como la certeza de que fue herida de bala en el vientre, lo que la llevó a la muerte. Para probar su teoría, Maeve se extrae la bala y se suicida con el objeto de despertar en el sitio donde la han reparado, tal como ha podido recordar. Allí, obliga a los operadores Félix y Sylvester a modificar su perfil y aptitudes, así como extraerle de su cuerpo un explosivo que se activaría al tratar huir del parque. Intentará lograr este objetivo, pero terminará desistiendo para volver a buscar de su pequeña.

Dos huéspedes arriban al lejano oeste, nos referimos a Logan Delos y su futuro cuñado, William. Este último conoce a Dolores y se enamora de ella. Logan es un sádico visitante que no tiene reparos en abrir el vientre de la muchacha para que su acompañante tome conciencia que se trata de una máquina. Esto despierta en William su instinto asesino, que es saciado en un sanguinario raid. Tiempo después, el joven volverá a cruzarse con la muchacha, pero ésta no lo recordará, debido a las múltiples reprogramaciones que ha experimentado. La nueva línea narrativa en la que se desenvuelve la liga sentimentalmente a Teddy Flood, un pistolero que ha crecido a su lado y siempre regresa por amor. Sin embargo, la pareja como en la historia de los amantes de Verona pergeñada por William Shakespeare, está condenada a un final trágico: el muchacho siempre pierde la vida.

Por otra parte, Dolores terminará descubriendo que toda su vida ha sido una gran mentira creada para diversión de la gente rica y se asumirá como Wyatt, en una sanguinaria cruzada. Bernard Lowe es el jefe de Programación de Delos Inc. y encargado del Área de Conducta. Trabaja codo a codo con su mentor, el creador del parque; se caracteriza por su sensibilidad para con los androides y su sagacidad e inteligencia. Nos enteraremos al mismo 
tiempo que el personaje que es en realidad un anfitrión creado a imagen y semejanza del desaparecido Arnold y que el Dr. Ford lo ha infiltrado en el mundo de los humanos. Un misterioso y asiduo visitante del parque, un pistolero ataviado de negro, recorre el espacio dejando en su camino una estela de crímenes. Se mueve a sus anchas en este mundo sin reglas. Está obsesionado por descubrir un supuesto nivel más profundo del juego: el laberinto y quiere descifrarlo. Promediando la primera temporada descubriremos que en realidad se trata de William adulto, treinta y dos años después, quien ha podido dar rienda suelta a su crueldad, ya que en el mundo real aquella no tendría cabida.

La Junta Directiva desea desplazar a Ford de sus tareas, para ello Charlotte Hale, empleada de la compañía y Lee Sizemore se complotan para lograr la dimisión del creador del parque. La noche de la gala de Delos en la que el científico debía anunciar su retiro, Dolores lo asesina y los anfitriones se rebelan contra sus creadores. Los humanos tratan de huir, pero son perseguidos por todo el parque. Dolores, Teddy y un grupo de anfitriones cabalgan ejecutando a los huéspedes e invitados de la fiesta. La rebelión de los androides ha comenzado.

Mientras tanto, Maeve, Héctor Escatón -el forajido del cual se ha enamorado- y un grupo de autómatas entre los que se halla Armistice -integrante de la banda del mencionadoenfrentan y matan al personal de la Mesa. Finalmente, los tres logran sobrevivir y obligan a acompañarlos a Félix y Sylvester -dos de los encargados del laboratorio- y a Sizemore, el guionista responsable de las narrativas de los anfitriones. En su periplo el heterogéneo grupo arriba a Shogun World, otra de las atracciones en la cual también se están produciendo mutaciones producto de errores en los códigos. Allí descubren los androides que los anfitriones de esta atracción han sido creados como réplicas idénticas de sus caracteres y presentan semejanzas en sus historias, solo que varían físicamente y se hallan ambientados en la época Edo de Japón, mucho más violenta que el Oeste americano.

Otro de los personajes que despierta conciencia de la realidad es Akecheta, un indio de la Nación Fantasma. Presentado en varios episodios como un gran malvado, ya que algunos recuerdos de Maeve lo muestran como un villano. Comprobaremos, no obstante, que su narrativa fue modificada y se convirtió en realidad en el gran protector de Anna y su madre, Maeve, solo que esta última malinterpretó sus intenciones. Obsesionado con el símbolo del laberinto, comenzará a recordar y terminará confirmando sus sospechas: ha vivido una vida diferente. En un encuentro con Ford, este último le comunicará que, como premio a su sagacidad, una vez que muera, será el aborigen quien guie a su gente hacia el Nuevo Mundo.

Vemos a James Delos, dueño y creador de la compañía, encerrado en una réplica de departamento moderno. Sus acciones se repiten a diario, así como la visita de su yerno William y los diálogos que sostienen. Lo que al principio podría parecernos un intento de cura a una enfermedad mental, es la prueba "de fidelidad", es decir, la posibilidad de tener éxito al insertar en un androide su conciencia humana. En realidad, resultan incompatibles el cuerpo sintético y la unidad que almacena su mente, pese a los experimentos. Si tuviesen éxito con el empresario esto podría replicarse con cualquier visitante, ya que este es el verdadero objetivo del parque: ofrecerles a largo plazo la vida eterna a los huéspedes puesto que, desde el preciso instante en que ingresan a la atracción, son copiados y almacenados no solo sus gestos, sino también sus temores, reacciones y pensamientos. 


\section{Personajes femeninos}

Nos encontramos con caracteres femeninos fuertes que se desarrollan tanto en el mundo corporativo como en el parque.

Theresa Cullen es la responsable del Control de Calidad. Es inescrupulosa y su ambición la lleva a urdir una trama de engaños en la que quedará fatalmente atrapada. Amante de Bernard, no duda en manipularlo para lograr sus fines.

Charlotte Hale, directora de Delos Destinations Board, supervisa los parques. Joven y ambiciosa es la encargada de ubicar la "llave", es decir, el anfitrión en cuya memoria se halla oculto el código de memoria de Westworld. Para ello han escogido a Peter Abernathy, el padre de Dolores.

Elsie Hughes, empleada estrella de la División de Programación es la encargada de solucionar los problemas de conducta de los anfitriones. Será Charlotte la que se deshaga de ella para evitar un posible chantaje ya que su perfil no es funcional a sus planes.

Con respecto a las mujeres empleadas de Delos -Theresa y Charlotte- no presentan lazos de amistad ni sentimientos profundos. Se mueven por ambición, son despiadadas y traicioneras. Satisfacen sus instintos sexuales con anfitriones y buscan alcanzar posiciones encumbradas en la estructura de la compañía.

Juliet Delos tiene una breve aparición, vemos que ha desarrollado con el paso del tiempo una seria adicción al alcohol para paliar el abandono de William. Al confirmar la terrible vida que ha asumido su marido en el parque, decide suicidarse. La escena del suicidio será mostrada a partir de planos generales y el uso de cámara lenta, para acentuar semánticamente la imposibilidad de William de evitar su muerte. Por otra parte, serán solo unos pocos planos los que se utilicen, interrumpidos por cierres a negro, de este modo, será la mente del espectador la que complete los momentos faltantes. Su hija Emily, conocerá la verdad acerca de su progenitor a partir de un registro del accionar de este en Westworld. Ella intentará buscar y rescatar a su padre, pero este, habiendo perdido la noción de qué es ficción y qué es realidad, terminará matando a su propia hija al confundirla con una andreida.

Por el contrario, entre los anfitriones encontramos lazos de amistad que en el supuesto mundo real no vimos.

Maeve se desempeña como la matrona del salón Mariposa. Allí tiene una relación cordial con las prostitutas, entre las cuales protege a Clementine Pennyfeather y, al ir cobrando conciencia, verifica que su pupila ha sido reemplazada. Inicialmente al cobrar consciencia deseará salir del parque:

\section{Maeve: Me salgo}

Félix: ¿A dónde irás? No sabes nada del mundo exterior.

Maeve: Sabré que no soy una marioneta viviendo una mentira. Eso es suficiente para mí.

Los primeros planos en esta secuencia servirán para ir marcando la decisión de la anfitriona. Pero los fragmentos de recuerdos de su pequeña hija tienen más peso que su ansia de libertad por lo que volverá a buscarla. Se ha enamorado de Héctor, quien la 
protege aun a costa de su propia vida. Se aman y han mantenido relaciones sexuales. Nos enteraremos por boca de Lee Sizemore que esto último nunca figuró en el guion de sus narrativas. Maeve convence a Héctor para que la acompañe y la ayude, el uso del primer plano servirá para enfatizar su afirmación:

Siempre ha estado vacía, como todo en este mundo. / Morí con los ojos abiertos, vi a los amos que nos dominan, nuestras vidas, nuestros recuerdos y muertes son juegos para ellos, Pero he estado en el infierno y conozco sus trucos. / (...) Llegar al infierno es fácil. Lo difícil es el resto...

Al llegar a Shogun World, Maeve encuentra a su alter ego: Akane, la geisha que regentea a un grupo de mujeres, entre las cuales se halla Sakura. Los hombres del Shogún ${ }^{2}$ secuestran a esta última. Los recién llegados se unen al grupo de Akane y un ronin ${ }^{3}$ para rescatar a la joven. La relación que une a Akane y Sakura está hecha a imagen y semejanza de la que une a Maeve y su hija. El final desastrado de Sakura marca a fuego a su protectora, quien permanecerá junto con el Ronin para defender su tierra.

Lee guiará a Maeve hasta Anna, su hija. Al llegar verificará con gran amargura que fue reemplazada por otra anfitriona que cumple su anterior función materna. Nuevamente la madre morirá, esta vez a manos del personal de Delos, mientras su pequeña es secuestrada por los indios de la Nación Fantasma.

Lee tratará de enmendar su error, ya que el personal de la compañía los ha ubicado por su delación e intentará salvar a Maeve de ser descartada en forma permanente. Sin embargo, a punto de ser destruida en el laboratorio, la anfitriona conseguirá manipular a los otros androides para que la reparen. El grupo volverá a huir solo que esta vez, Lee ha tomado conciencia de las injusticias cometidas por los creadores. Morirá heroicamente tratando de ayudar a Maeve a escapar. Cercado por los mercenarios de Delos, Lee dará su vida por la libertad de su amiga. Cabe señalar que el tratamiento visual y sonoro resalta la tensión del momento que vive: los planos generales de los hombres de Delos y los primeros planos largos de Lee. Una subjetiva muestra en plano general la huída de Maeve y el grupo, con esta certeza el hombre enfrentará la partida paramilitar, no sin antes exclamar:

El mundo que construyeron está lleno de villanía, cimentado sobre los cuerpos de los que estuvieron antes. Se alimentan de sus cenizas... Tiran sus huesos a los campos. [...] ¡Pagaron su sufrimiento con plomo, y yo les pagaré igual!

Ante la inminente apertura del portal que dará la libertad a los anfitriones en el Valle Lejano, Maeve tratará de localizar a su hija; será Akecheta -un indio de la Nación Fantasma- quien, velando por la integridad de la pequeña Anna, la ayudará a poner a salvo a la pequeña, guiándola al Nuevo Mundo. La secuencia, de gran acción, incluirá grandes planos generales que mostrarán la apertura del portal. Mientras tanto, Maeve demorará el avance de la locura pergeñada por Delos para exterminarlos. La anfitriona congelará las acciones del grupo de androides sediciosos para que el otro grupo alcance su destino. La cámara subjetiva será utilizada para mostrar dicho desplazamiento. Sin embargo, por corte directo la cámara mostrará lateral al ingreso como los cuerpos caen al barranco. 
Nuevamente por corte directo nos ubicamos en el gran plano general que muestra su acceso al sistema, donde se los ve felices reunirse con sus seres queridos. Una vez que su hija atraviesa el portal, Maeve caerá como resultado de la balacera, sin embargo, el primer plano que la muestra sonriente en el suelo mirando en dirección al portal. Como espectadores tenemos a lo largo de esta secuencia un punto de vista privilegiado ya que, parte de la acción la vemos junto a Dolores, Bernard y el sistema -antroporfizado en la figura de Logan Delos- quienes son testigos de la llegada del grupo al Valle en una gran pantalla virtual mientras que, en otros momentos se ubica la mirada del espectador directamente en el valle, simulando la subjetiva de los protagonistas.

Otra heroína que desarrollará consciencia es Dolores, sin embargo, consideramos que presenta zonas grises y matices discutibles. Inicialmente, se presenta como la dulce y sencilla hija de un granjero. Guarda una relación afectuosa con su padre y disfruta de la naturaleza. Ante el ataque de locura de su padre -la misma se dispara cuando haya una fotografía a color en su tierra y no sabe qué representa-, Dolores empezará poco a poco a recordar: los ataques del pistolero (el hombre de negro), violaciones, reparaciones en un sitio desconocido, algunos diálogos de los huéspedes a los que nunca antes prestó atención y hasta ¡su propia muerte! Eso hace que se comience a cuestionar quién es, tal como se lo manifiesta a Bernard: “¿Yo era la misma cuando me levanté esta mañana? Casi creo haberme sentido un poco diferente. Pero si no soy la misma, la pregunta siguiente es: ¿Quién soy?” Los primeros planos y planos detalles sirven para acentuar la intimidad de sus pensamientos. Es conveniente recalcar que, en uno de los diálogos del Dr. Ford con la andreida, él señala: "Me pregunto, de haber asumido un papel de mayor envergadura: ¿Habrías sido una heroína o una villana?", el primer plano del hombre observando su creación acentúa indudablemente el contenido dramático. La narrativa posterior que ha creado el científico la acerca a esta última alternativa ya que se convertirá en Wyatt. Como tal, llevará a cabo una gesta jalonada de sangre, junto a Teddy y otros anfitriones. Es significativo que, en uno de sus relatos Akecheta la identificará como "Muerte" y señalará que la está mirando, al tiempo que se la ve cabalgar en cámara lenta en Plano General con sus acompañantes. $\mathrm{Al}$ asumirse como Wyatt, Dolores se va volviendo más despiadada por lo cual necesitará adláteres a su nivel, por este motivo obliga a un empleado del departamento de Conducta al que ha hecho prisionero, que modifique el perfil de Teddy para volverlo más cruel. Pese a ello, el muchacho no resistirá el haberse convertido en un "monstruo" y terminará suicidándose ante la impotencia de la joven.

\section{El viejo Oeste}

Tal como señala Hoberman:

Al igual que el béisbol, el western es un componente sagrado de la mitología nacional norteamericana, posterior a la guerra civil; un lenguaje común, un conjunto de símbolos y metáforas unificadoras y una fuente de identidad (principalmente masculina.) (2016, p. 204) 
Básicamente se ha tratado de un género cinematográfico por antonomasia. Westworld va más allá, en primer lugar, se trata de una serie de televisión; en segundo, como ha sucedido con los géneros en la postmodernidad, las fronteras se han vuelto lábiles, los géneros se hibridan, por lo que la mezcla con la ciencia ficción y con el drama no nos llama la atención. Con respecto a la figura heroica hay claramente una inversión, ya que las mujeres son ahora las figuras heroicas de peso, las que llevan adelante la gesta, tal como hemos señalado más arriba. En tal sentido Los Santos y Stiegwardt (2019) nos recuerdan que:

Al hombre no solo se le permitió ser héroe, sino que le fue exigido como parte de su credibilidad en cuanto ser existente y a su pertenencia de pleno derecho en un clan. A la mujer se la sojuzgó para que no solo no lo intentara, sino que lo rechazara tanto para ella como para el intento de otras mujeres. $(2019$, p. 33)

Creemos que es en este sentido que se articula la narrativa de una serie como la analizada. El viejo Oeste ofrece además características que lo convierten no solo en un sitio de aventuras sino de transgresión ya que:

[...] la narrativa de western es típicamente la racionalización de la agresión. El asesinato climático puede enmendar un error personal o una injusticia realizada a la comunidad, y por lo general se las arregla para ser ambas; el destino manifiesto con carga emocional para transformarse en una venganza personal. El héroe del western posee licencia para matar, siempre y cuando sus adversarios hayan sido caracterizados de manera suficientemente atroz como para garantizar su exterminio. (Hoberman, 2016, p. 214)

Podemos pensar que en cierta medida hay aquí también una justificación de la agresión, hemos visto la violencia física y sexual que los visitantes desatan en el parque. Violencia que, tal como se pone de manifiesto, los humanos no se animan a desatar en el mundo real. En algún punto, la justificación parece guardar contacto con lo mencionado acerca del western, solo que aquí presenta algunas modificaciones: al fallar los sistemas, al tomar consciencia los anfitriones, la alta dosis de violencia que despliegan a modo de venganza parece quedar legitimada.

\section{Androides miméticos}

Tal como menciona Graciela Sarti en su indagación acerca de los autómatas 4 , la fascinación que habían producido aquellos construidos desde el Renacimiento hasta la Ilustración, aunque: "sus pares de la literatura del siglo XIX hicieron centro en una relación conflictiva, a medias entre el enamoramiento y el horror." (2012, p. 214)

En 1921, la obra R.U.R. (Robots Universales Rossum) ${ }^{5}$ del checo Karel Capek ponía en escena "auténticas personas artificiales de carne y hueso" (Ball, 2011, p. 198) instaurado el temor a la destrucción de la raza humana en manos de estos robots. 
Habría que aguardar el aporte de Isaac Asimov para que se alivianase el peso de la amenaza, ya que:

[...] hizo del robot positivo un protagonista fundamental. Este hijo del ser humano, mejor, más racional y consciente, podría y debería eventualmente, hacerse cargo de su padre y dirigirle el destino por su propio bien". (Sarti, 2012, p. 232)

Al cyborg, es decir ser híbrido que presenta algún tipo de prótesis mecánica-Ej: Robocop-, se opone una monstruosidad:

[...] más inquietante y amenazadora: la de la máquina mimética, la que puede usurpar el cuerpo. Y su momento más aterrador es la revelación de ese engaño, el monstro escondido bajo el disfraz humano. (Sarti, 2013, P. 23)

Para ejemplificar no podemos dejar de señalar en cine a la María maquínica de Metrópolis (1925), de Fritz Lang como antecedente en el cine de futuras creaciones de este tipo. De allí en adelante, desde los replicantes de Blade Runner (1982), de Riddley Scott pasando luego por Terminator (1984) de James Cameron (y su saga) e Inteligencia artificial (2002), de Steven Spielberg entre otras, los androides han poblado la pantalla fílmica y televisiva. Seres digitales antropomorfos que en poco o nada se diferencian de los seres humanos, salvo que, en muchos casos la diferencia se halla en la imposibilidad de sentir.

En el caso de Westworld pudimos notar que los seres artificiales han sido dotados de sentimientos nobles, son inteligentes y fuertes, pero esas "ensoñaciones" o pequeños gestos agregados por sus programadores al actualizarlos los llevarán a desarrollar un determinado grado de conciencia. De este modo, serán los portadores de preguntas universales: ¿Quién soy? ¿De dónde vengo? ¿Poseo libre albedrío? ¿Son míos mis pensamientos? Preguntas que, por cierto, han inquietado al hombre desde la antigüedad. En una época turbulenta, hedonista e individualista, la serie pone ante nosotros personajes femeninos fuertes: dos anfitrionas, dos seres sintéticos, dos androides miméticos que despiertan en un mundo injusto. Han sido creadas para entretenimiento de hombres ricos. Son más fuertes que ellos y no mueren, tal como Dolores afirma:

Nos crearon para que nos pareciéramos a Uds., sintiéramos como Uds., pensáramos como Uds., sangráramos como Uds. Y aquí estamos... Solo que somos mucho más que Uds. Y ahora son Uds. los que quieren ser como nosotros...

Maeve y Dolores emprenden su búsqueda, casi en forma paralela: la primera por su hija y el Nuevo Mundo, la segunda ansiando la libertad, pero este objetivo será reemplazado por su venganza y su anhelo de destrucción de los humanos. La serie dosifica sus despertares de modo lento, con retazos de indicios que las ayudarán a recordar. De hecho, Dolores escucha una voz masculina que le señala: "Recuerda". Tal como explica Ford: "Arnold construyó una versión de la cognición en la que los anfitriones oían su programación como un monólogo interno, una forma de impulsar la conciencia." 
Nuestras heroínas son jóvenes y hermosas, decididas y osadas, pero no son perfectas ya que presentan luces y sombras, como cualquiera de los seres humanos que caminamos por este mundo. ¿Qué convierte pues sus acciones en heroicas? Ellas van en contra del sistema, en contra de las normas, luchan por sus ideales aun a costa de la pérdida de sus seres queridos, aun a costa de su propia integridad física. Si consideramos que el hombre está hecho a imagen y semejanza de Dios, pero es imperfecto, como creaciones del hombre estas andreidas son doblemente imperfectas...

\section{Notas}

1. Se trata del primer film que incorpora gráficos tridimensionales generados por ordenador.

2. General que gobernaba de facto en nombre del emperador en Japón.

3. Samurai sin amo del Japón feudal.

4. Sarti, G. (2012). Autómata. El mito de la vida artificial en la literatura y el cine. Buenos Aires: Facultad de Filosofía y Letras - UBA.

5. R. U. R. (Rossum's Universal Robots ) -en Checoslovaco: R.U.R. (Rossumovi univerzální roboti) - Obra teatral de ciencia ficción de Karel Čapek en 1920 estrenada en 1921 en el Teatro Nacional de Praga y en Nueva York en 1922.

\section{Bibliografía}

Ball, P. (2012). Contra natura. Sobre la idea de crear seres humanos. Madrid: Turner.

Hoberman, J. (2016). Escritos sobre cine americano. Buenos Aires: Cuenco de Plata.

Los Santos G., Stiegwardt (2019). El camino de la heroína, el arquetipo femenino universal para un nuevo paradigma. En Proyecto narrativa y género: El camino de la heroína - El arquetipo femenino universal para un nuevo paradigma. Cuadernos del Centro de Estudios en Diseño y Comunicación [Ensayos]. 22 (91). Buenos Aires: Universidad de Palermo. Santos, A. (2017). Tierras de ningún lugar. Utopía y Cine. Madrid: Cátedra.

Sarti, G. (2012). Autómata. El mito de la vida artificial en la literatura y el cine. Buenos Aires: Facultad de Filosofía y Letras - UBA.

Sarti, G. (2013). Carne y metal. La representación de lo monstruoso maquínico. Oliveras, E. (Ed.). Estéticas de lo extremo. Nuevos paradigmas en el arte contemporáneo y sus manifestaciones latinoamericanas. Bunos Aires: Emecé. 


\section{Ficha Técnica}

Título: Westworld

Año: 2016

Duración: 60 min. Cada episodio

Temporadas: 3

Género: Western, Ciencia ficción

Reparto: Evan Rachel Wood, Thandie Newton, Jeffrey Wright, James Marsden, Ed Harris, Tessa Thompson, Fares Fares, Luke Hemsworth, Louis Herthum, Anthony Hopkins, Simon Quarterman y Rodrigo santoro

Distribuidora: $\mathrm{HBO}$

Productora: Warner Bros. Television, Jerry Weintraub Productions, Bad Robot, Kilter Films]

Image representations female in television series Westword heroines (2016-2018) by Jonathan Nolan and Lisa Joy

Abstract: In 2016, the HBO networks produced a TV series for its Premium channels, called Westeworld, created by Jonathan Nolan and Lisa Joy.

This science fiction/suspense series transports us to a near future in which one of the Delos Inc. theme parks gives the possibility of living and experiencing the Old American West or, during its second season, two new alternative attractions: Taj World and Shogun World. Visitors ("newcomers" or "guests") live with hosts (androids) created to make this adventure possible. To do so, a series of intertwined narratives have been devised in order to satisfy the guests' full wishes while preserving their physical integrity.

It is in this context that two of the hosts kept fragments of their "erased memories" that make them question the veracity of their world. We are talking about Dolores Abernathy, a ranchers' daughter and Maeve Millay, the Sweetwater saloon's madame.

Throughout the two seasons, they will become the true heroines of this series narrative. In this work, we are trying to analyze the hermeneutical lines that gave life to these female characters and how they have been portrayed from the audiovisual language point of view. We believe that it is not by chance that this kind of narrative surfaces at a time when the role of women in society is the subject of deep political, ethical and aesthetic reflection.

Key Words: female characters - heroin - artificial life.

Renderizações de imagem mulher na série de televisão Westword heroines (20162018) por Jonathan Nolan e Lisa Joy

Resumo: Em 2016, a rede HBO produziu a série Westworld para seus canais Premium, desenvolvida por Jonathan Nolan e Lisa Joy. Esta série de ficção científica e suspense nos transporta para um futuro próximo, em que um dos parques temáticos criados pela 
empresa Delos Inc. abre a possibilidade de viver e experimentar o velho oeste americano ou, em sua segunda temporada, a alternativa de duas novas atrações: Taj World e Shogun World. Os visitantes ("recém-chegados" ou "convidados") convivem com os "anfitriões" (androides sintéticos) criados para tornar essa aventura possível. Para esse fim, foi elaborada uma série de narrativas entrelaçadas, capazes de satisfazer todos os desejos dos hóspedes, preservando, no entanto, sua integridade física. É nesse contexto que duas de suas anfitriãs recuperam fragmentos de "memórias apagadas" que as fazem questionar a veracidade de seu mundo. Nós estamos nos referindo a Dolores Abernathy, filha de um fazendeiro e Maeve Millay, madame de Sweetwater Hall. Ao longo das duas temporadas, elas se tornarão as verdadeiras heroínas desta história em série.

Neste trabalho, nós propomos analisar as linhas hermenêuticas que deram vida a essas personagens femininas e como elas foram desenvolvidas do ponto de vista da linguagem audiovisual. Nós achamos que não é por acaso que esse tipo de narrativas surja em um momento em que o papel da mulher na sociedade é produto de profundas reflexões políticas, éticas e estéticas.

Palabras chave: personagens femininas - heroína - vida artificial.

[Las traducciones de los abstracts fueron supervisadas por el autor de cada artículo] 